\title{
Contribution to the study of the perception of the risk of water-related diseases in Abéché
}

\section{Contribution à l'étude de la perception du risque des maladies liés à l'eauà Abéché}

\author{
Dombor Djikoloum Dingao ${ }^{1}$, Tidjani Assouni ${ }^{2}$, Adimatcho Aloua ${ }^{3}$, Tchiadeu Gratien ${ }^{4}$
}

\author{
${ }^{1}$ Ecole Normale Supérieure d'Abéché \\ dddombor@gmail.com \\ ${ }^{2}$ Université Adam Barka d'Abéché, \\ ${ }^{3}$ Ecole Normale Supérieure d'Abéché \\ ${ }^{4}$ Université de Douala
}

Received: 28 Nov 2021; Received in revised form: 21 Jan 2022; Accepted: 03 Feb 2022; Available online: 08 Feb 2022 C 2022 The Author(s). Published by Infogain Publication. This is an open access article under the CC BY license (https://creativecommons.org/licenses/by/4.0/).

\begin{abstract}
Vulnerability is a combination of factors, damage and social response. The latter depends on the perception of the exposed population. The perception of the phenomena and the risks incurred, their causes and their consequences leads the inhabitants to expose themselves to these threats. This contribution analyzes the behavior of the Abéche's population according to their perception of the health risk associated with water. To achieve the objectives set, a focus group was carried out with 250 and a household survey with 1516 households distributed differently by district. It follows that knowledge, perceptions and attitudes in the event of morbid episodes highlight a major paradox. The study population is characterized by very heterogeneous representations and attitudes in matters of health. Contrary to simplistic or ideologically marked interpretations, the perception of water-related diseases is primarily based on lessons learned from deep experience of its medical and epidemiological characteristics. However, these diseases remain poorly understood in Abéché. Despite their major impact on morbidity and mortality, the perception of their causes, manifestations and treatments is most often approximate and is the subject of multiple amalgamations.
\end{abstract}

Keywords-Perception, Risk, Water-related diseases, Vulnerability, Abéché

Résumé- La vulnérabilité est une combinaison des facteurs, de l'endommagement et de la réponse sociale. Cette dernière est tributaire de la perception de la population exposée. La perception des phénomènes et des risques encourus, de leurs causes et de leurs conséquences conduit les habitants à s'exposer à ces menaces. Cette contribution analyse les comportements de la population abéchoise en fonction de leur perception du risque sanitaire lié à l'eau. Pour atteindre les objectifs fixés, un focus groupe a été fait avec 250 et une enquête ménage avec 1516 ménages répartis différemment par quartier. Il en résulte que les connaissances, les perceptions et les attitudes en cas des épisodes morbides mettent en évidence un paradoxe majeur. La population d'étude se caractérise par des représentations et des attitudes en matière de santé très hétérogènes. A l'encontre d'interprétations simplistes ou idéologiquement marquées, la perception des maladies liées à l'eau reposent avant tout sur les enseignements d'une profonde expérience de ses caractéristiques médicales et épidémiologiques. 
Cependant, ces maladies restent mal connue à Abéché. En dépit de leur incidence majeure sur la morbidité et la mortalité, la perception de leurs causes, de leurs manifestations et de leurs traitements est le plus souvent approximative et fait l'objet de multiples amalgames.

Mots clés-Perception, Risque, Maladies liées à l'eau, Vulnérabilité, Abéché

\section{INTRODUCTION}

L'état de santé d'un individu est la résultante d'un système multifactoriel complexe (Amat-Roze J.M., 1984). La perception d'un risque sanitaire est relative et celle d'un besoin de santé, ambiguë. Malgré l'élaboration scientifique de normes sanitaires, être en bonne santé est une appréciation éminemment variable, où se mêlent des éléments physiologiques, psychologiques, sociaux, culturels, du ressort de l'individu mais aussi du collectif et du politique. Ainsi, les savoirs, les croyances, les valeurs d'une société déterminent des perceptions originales des risques pathogènes, des états morbides et des remèdes (Amat-Roze J.M., 1984). En découlent des stratégies culturelles porteuses de comportements aggravant, favorisant ou atténuant, éliminant un risque sanitaire. Ces pratiques façonnent alors de véritables aires culturelles des maladies. De ce fait, l'ignorance de ces logiques élève des barrières d'incompréhension et compromet la réussite des politiques de santé destinées à l'amélioration de l'état de santé des populations. La perception du risque sanitaire induit le processus décisionnel qui conduit les habitants à adopter des comportements. L'analyse des causes profondes de vulnérabilité des communautés est basée sur une démonstration de la perception des phénomènes et des risques encourus, de leurs causes et de leurs conséquences, qui conduit les habitants à s'exposer à ces menaces. Des contraintes structurelles sous-jacentes d'ordre structurel, social, culturel, économique et politique, indépendantes de l'aléa et ancrées dans le quotidien, les poussent à ces comportements. Cette communication se donne d'analyser les comportements de la population abéchoise en fonction de leur perception du risque sanitaire lié à l'eau et les contraintes qui y sont liées.

\section{METHODOLOGIE}

\subsection{Présentation de la zone d'étude}

La ville d'Abéché (figure 1) est située entre les $13^{\text {ème }}$ et $14^{\text {ème }}$ degré de latitude Nord et les $20^{\text {ème }}$ et $21^{\text {ème }}$ degré de longitude Est. Elle est limitée au Nord par la Sous préfecture de Bourtaye, au Sud par celle d'Abgoudame, à l'Est par la Sous-préfecture d'Amleyouma et à l'Ouest par celle de Guéri. Elle connait une dynamique spatiodémographique remarquable depuis ces quinze dernières années. Sa population était évaluée selon le RGPH21 de 2009 à 138.684 habitants dont 65.483 femmes et 73.201 hommes et est passée en 2014 à 166.757 habitants $(78.738$ femmes et 88.019 hommes), en 2015 à 172.746 habitants et en 2016 à 178.896 habitants.

Par ailleurs, Abéché connait un climat de type sahélien, caractérisé par une saison des pluies qui s'étale sur trois mois (juillet à septembre) et une saison sèche qui s'étend sur neuf mois (octobre à juin); des précipitations relativement faibles, variant entre 500 et $600 \mathrm{~mm}$ par an ; et une température moyenne annuelle de $32^{\circ} \mathrm{C}$ (D. D. Dombor et al, p. 96). Elle repose sur un socle granitique, rendant difficile tout aménagement hydraulique, compromettant un accès durable à l'eau potable.

${ }^{1} \mathrm{RGPH}$ : Recensement Général de la Population et de l'Habitat 


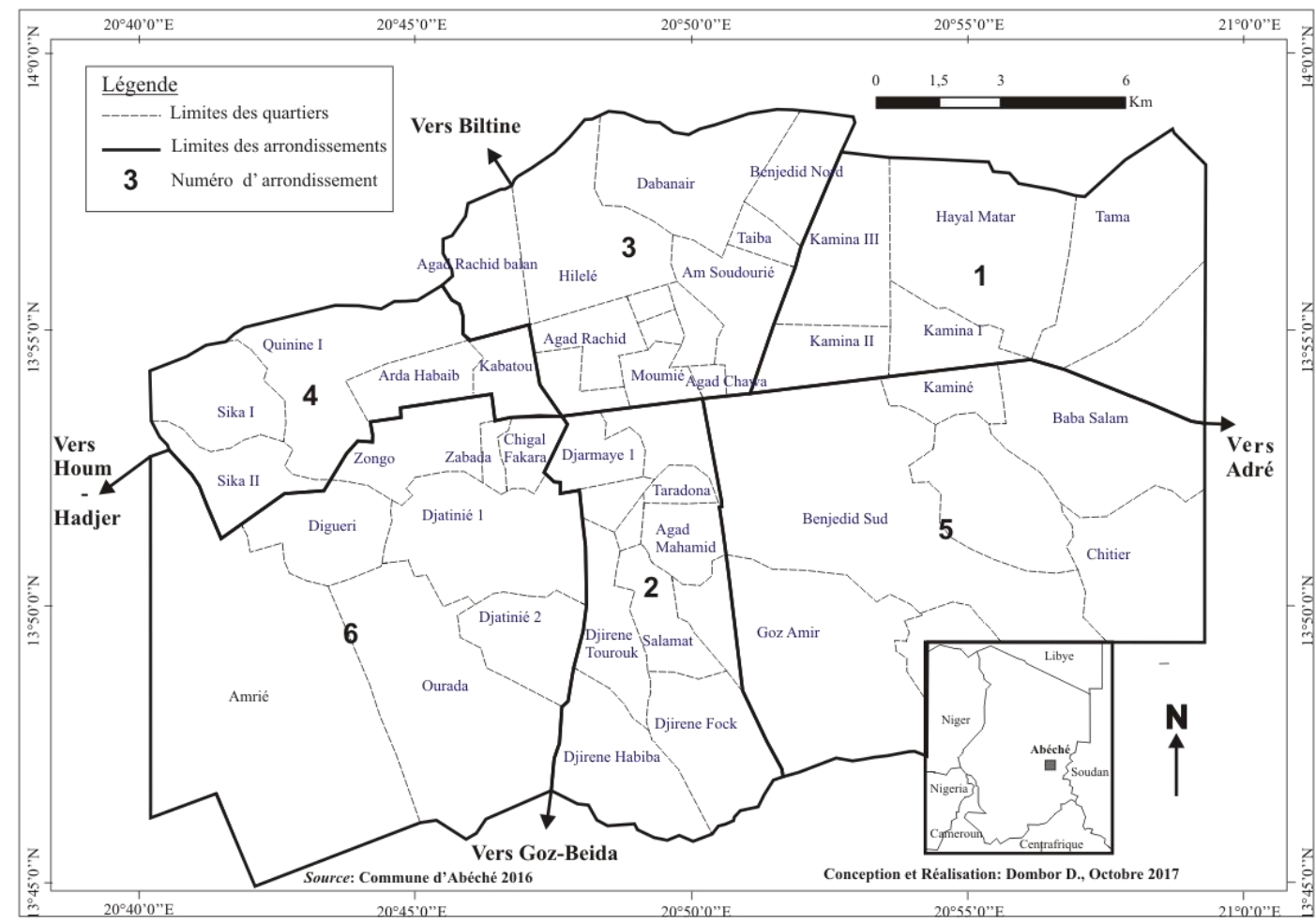

L'étalement urbain qui caractérise la ville d'Abéché depuis une dizaine d'années a entravé une vision globale de la ville et, en particulier, du circuit de l'eau. Ainsi comme le dit Catherine Baron (2006) ceci a un impact sur la desserte en eau de certains quartiers, notamment de ces espaces urbaines qualifiées, selon les époques et les lieux, de périphériques, de bidonvilles, d'irréguliers. En effet, face à la croissance urbaine exceptionnelle qu'a connue Abéché, une part croissante de la populationse trouve exclue de l'accès à une eau potable.

\subsection{Méthode}

Pour cette étude, l'analyse systémique et les méthodes a posteriori, sont utilisées. Dans l'optique d'atteindre la population visée, un certain nombre de ressources humaines indispensables et capables de fournir des renseignements ou des informations utiles à la vérification des hypothèses et à l'atteinte des objectifs de recherche étaient identifiées. Ainsi la population d'étude est constituée des groupes de femmes qui sont reconnues comme les principales actrices dans tout projet d'approvisionnement en eau et d'assainissement (Banque Mondiale, 1999) et des chefs de ménages qui sont les décideurs au sein des ménages. Pour une collecte optimale des informations, la taille de l'échantillon a été estimée à l'aide du logiciel Epi-Info avec un intervalle de confiance de $95 \%$ sur la base de données fournie par la Commune d'Abéché. Cette méthode a permis de déterminer un échantillon de 1516 ménages répartis différemment par quartier. Les ménages interrogés ont été retenus sur la base d'un échantillonnage aléatoire simple. Un focus group avec 250 femmes a été fait.

L'enquête s'est déroulée après une formation du personnel d'enquête (18 au total). Les enquêteurs ont été formés à travers un guide approprié d'une trentaine de pages. Ce guide a été spécialement conçu pour que les enquêteurs aient la même démarche et que les informations soient recueillies sans biais.

\section{RESULTATS ET DISCUSSIONS}

2.1- Perception de l'eau par les populations à Abéché

Les populations abéchoises sont conscientes des périodes de rareté et d'abondance de l'eau, de sa pollution et de sa dangerosité pour la santé. L'analyse de la connaissance générale de l'eau de la population à l'aide d'indicateurs liés à la représentation de son essencemême et de son origine géographique permet de positionner la population par rapport à son appréhension des problèmes étudiés dans le temps et dans l'espace. Leurs pratiques quotidiennes en temps de pénurie, d'abondance et leurs connaissances des moyens de protection face aux dangers, les exposent à la menace ou l'aggravent.

Dans la ville d'Abéché, $100 \%$ des ménages enquêtés (tableau 1) considèrent l'eau comme utile pour boire, se laver, nettoyer et cuisiner (à), ou bien comme 
source de revenus par le commerce $(23,5 \%$ des ménages enquêtés). Pour cette population, l'eau permet de satisfaire les besoins quotidiens élémentaires. La représentation de l'eau comme étant liée à certaines maladies est citée par $53 \%$ des enquêtés et $35 \%$ des groupes des femmes (focus groupe). $46 \%$ des répondants pensent que les eaux polluées et stagnantes apportent des maladies comme le paludisme et le choléra.

Tableau 1. Représentations de l'eau par la population

\begin{tabular}{|l|l|l|l|}
\hline Représentation de l'eau & $\begin{array}{l}\text { Enquêtes ménages (\% } \\
\text { des observations) }\end{array}$ & $\begin{array}{l}\text { Focus groupes avec les } \\
\text { femmes (\% des } \\
\text { observations) }\end{array}$ & Moyenne (\%) \\
\hline Besoins élémentaires & 100 & 100 & 100 \\
\hline Eaux polluées et maladies & 17 & 10 & 13,5 \\
\hline Eaux stagnantes et maladies & 11 & 8 & 9,5 \\
\hline Manque d'eau, achat nécessaire & 93 & 72 & 82,5 \\
\hline Importante pour la vie & 100 & 100 & 100 \\
\hline Eau source de conflit & 23 & 48 & 35,5 \\
\hline Eau source de revenus & 23,5 & 21 & 22,25 \\
\hline
\end{tabular}

Source : enquête ménage Mars à Octobre 2016

Les évocations de l'eau comme source importante pour la vie sont à $100 \%$. $23 \%$ des répondants des enquêtes ménages et $48 \%$ des femmes considèrent l'eau comme source de conflit. La part des femmes est plus élevée que celle des chefs de ménage, par ce que les femmes font partie des personnes chargées de la collecte de l'eau
Le tableau 2 montre la perception de la population de la nature de l'eau. Plus de $60 \%$, soit $72,5 \%$ de la population de tous les quartiers adhèrent concernant sur la nature de l'eau, à l'hypothèse d'une nature divine au détriment d'une nature physique qui n'est que secondairement évoquée. Pour cette population la ressource en eau dépend de la volonté de Dieu.

Tableau 2. Perception de la population de la nature de l'eau

\begin{tabular}{|l|l|l|l|}
\hline Nature de l'eau & $\begin{array}{l}\text { Enquête ménages } \\
(\boldsymbol{\%})\end{array}$ & Focus groupes (\%) & Moyenne (\%) \\
\hline Don de Dieu & 63 & 82 & 72,5 \\
\hline Ressource naturelle & 18 & 6 & 12 \\
\hline Divine et naturelle & 19 & 12 & 15,5 \\
\hline Ni l'un, ni l'autre & 0 & 0 & 0 \\
\hline total & 100 & 100 & 100 \\
\hline
\end{tabular}

Source : enquêtes par questionnaire réalisées entre mars et octobre 2016.

Cette unanimité des réponses repose certainement sur les habitudes populaires d'attribuer dans le langage une essence divine aux «bonnes choses » sans pour autant se représenter une création divine de la chose. D'autres résultats corroborent également l'importance vitale de l'eau puisque $77,5 \%$ des répondants la considèrent comme « indispensable » plutôt qu' « utile ».

En revanche, les habitants enquêtés ne considèrent pas pouvoir jouir sans contraintes ni restriction de l'eau.
En effet, le manque d'eau pour les besoins quotidiens est ressenti de manière majoritaire dans la ville (pour 65,5\% des répondants des enquêtes ménages et $72.6 \%$ seulement) surtout les quartiers dont les habitants puisent principalement l'eau du sous-sol librement (puits) et qui sont dépendants de systèmes d'approvisionnement extérieurs. Cependant, si le problème du manque d'eau apparait prioritaire pour plus de $70 \%$ des habitants de la ville, ceux des quartiers utilisant les forages n'ont pas 
d'idée sur les problèmes du manque d'eau.

En ce qui concerne son origine, 54,7 \% des répondants ont conscience d'une origine naturelle de l'eau venant de l'amont, et 45,3\% pensent que l'eau a une origine soit divine soit au contraire évoquent une origine plus pragmatique (l'eau vient du robinet, de la pompe ou du puits dont ils se servent au quotidien).

Les habitants des quartiers informels sondés ont globalement un discours qui traduit une conscience de la dualité face à l'eau : entre excès d'eau et manque d'eau, entre la nécessité de l'eau source de vie et l'eau source de dangers. $39 \%$ des répondants considèrent en effet l'eau à la fois comme un bienfait et un danger, même si globalement ils associent l'eau principalement aux bienfaits qu'elle procure plutôt qu'aux dangers qui lui sont liés.

Les populations abéchoises ne considèrent pas l'eau comme une ressource inépuisable ou un robinet ouvert. Elles ont conscience à la fois de la rareté de l'eau, de ses origines naturelles et de sa destination dans leur espace de vie, et des problèmes environnementaux qu'elle peut susciter. Ce qui semble les préoccuper en priorité est le manque d'eau potable, ce qui explique pourquoi ils considèrent l'eau avant tout comme un élément essentiel à leur survie, un moyen de satisfaire leurs besoins quotidiens.

\section{2- $\quad$ Perception des risques sanitaires liés à l'eau et des moyens de protection}

\subsection{1- Perception de la menace sanitaire liée aux ordures ménagères}

Répondant aux questions sur les menaces liées à la présence des ordures ménagères, $87 \%$ des répondants citent le paludisme, $18,4 \%$ les problèmes de peau et $37 \%$ les maladies intestinales. Globalement la perception de la menace sanitaire liée à la pollution de l'environnement est ainsi élevée et homogène entre les arrondissements.

La figure 2 montre que le paludisme et les maladies respiratoires sont déclarés par la population comme les maladies récurrentes liées à l'insalubrité. Dans tous les arrondissements le paludisme est la maladie la plus déclarée. Dans le 2eme et le 5eme arrondissement les répondants considèrent les maladies respiratoires comme deuxièmes risque sanitaire après le paludisme.

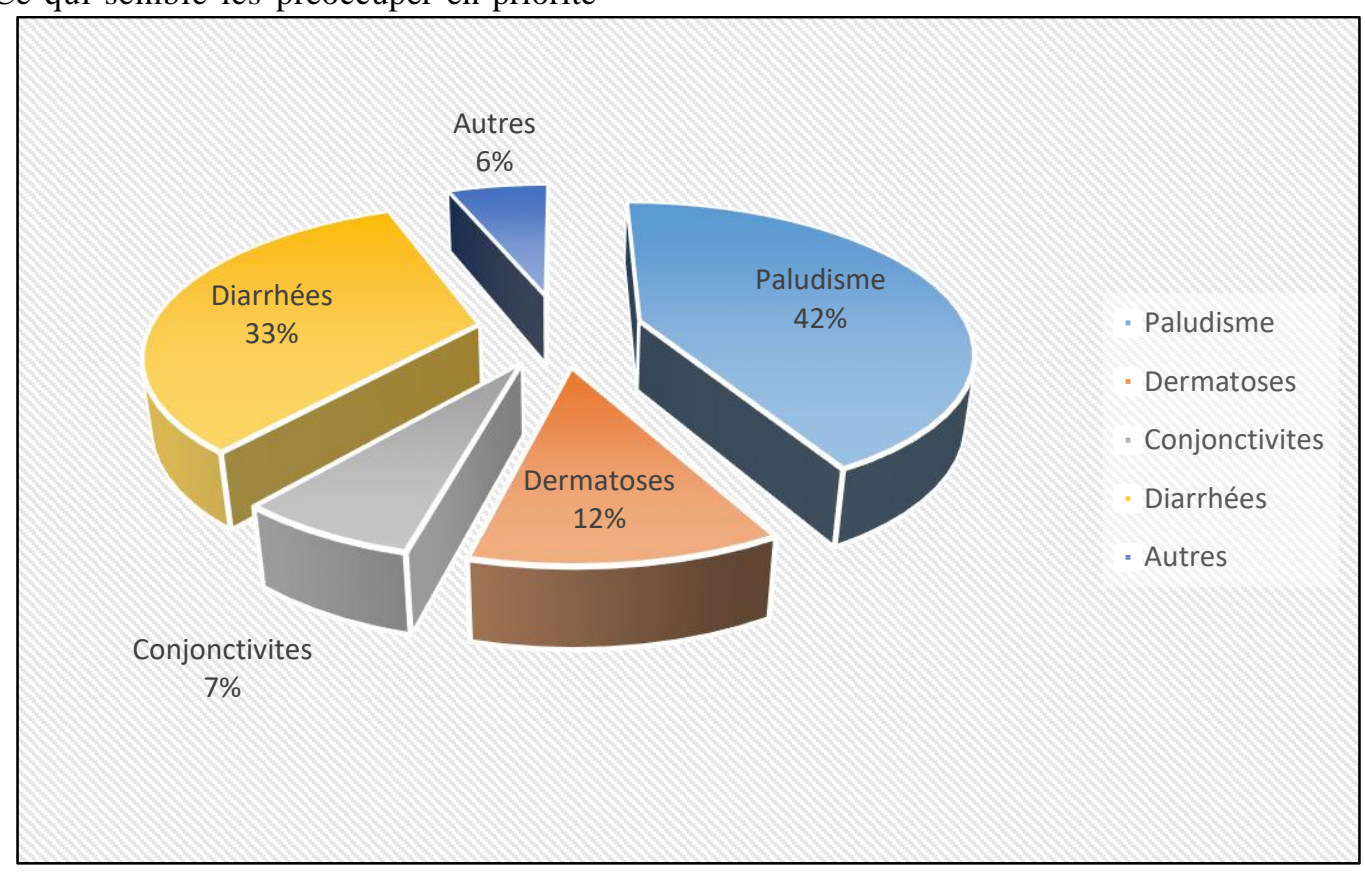

Figure 1. Perception des dangers liés aux ordures ménagères.

Source : enquêtes ménages 2016

\subsection{2- Perception de la menace sanitaire liée au contact avec l'eau}

Près de $54 \%$ des répondants citent la typhoïde, les démangeaisons et les maladies de peau comme conséquence potentielle d'un contact avec de l'eau souillée. Seulement $27,7 \%$ des répondants ignorent le risquesanitaire en se lavant ou se baignant dans de l'eau polluée, et seulement $18,3 \%$ des répondants se trompent en citant le paludisme comme conséquence.

Si $82 \%$ des répondants reconnaissent que les enfants se baignent régulièrement dans les cours d'eau et les eaux stagnantes, plus de deux tiers d'entre eux condamnent cette pratique. En effet, les cours d'eau et les marres, pour 
près de $70 \%$ des habitants sondés, sont des zones de décharge et d'évacuation des eaux usées, des espaces dangereux. Ils considèrent ces espaces comme pollués et sales. Néanmoins les habitants à proximité de cette zone et ou habitués à utiliser ces sources et à la pratique de la baignade, se sentent moins menacés par les maladies relatives que ceux des autres endroits. Notons que les cours d'eau et les marécages sont considérés comme des espaces utiles aux lavages. La moitié seulement des répondants considère d'ailleurs que les cours d'eau et les marécages sont toujours sales.

Les répondants qui se lavent ou utilisent régulièrement l'eau des cours d'eau et des marécages citent moins de maladies liées à ses sources que les autres, et $22 \%$ d'entre eux, contre moins de $12 \%$ pour les autres répondants, pensent qu'utiliser l'eau de ces sources ne représente aucune menace particulière. L'explication de ce comportement repose en partie sur le peu de considération portée aux maladies de peau, très fréquentes, bénignes et ne faisant généralement l'objet d'aucun traitement.

Malgré ces petites nuances entre les réponses, globalement, les répondants de tous les quartiers ont tout à fait conscience des risques liés à la baignade en citant de nombreuses maladies, tandis que globalement seulement 3,6 \% des répondants pensent qu'il n'y a aucune conséquence négative à cela.Ainsi, la perception élevée des menaces sanitaires liées à l'utilisation des eaux polluées n'explique pas les pratiques dangereuses observées sur le terrain.

\subsection{3- Perception de la menace sanitaire liée à la pollution de l'eau}

Les populations abéchoises se fient à des critères de potabilité liés à l'aspect physique de l'eau : sa couleur, sa transparence, son odeur et son goût. Pour la potabilisation de l'eau, les habitants pratiquent l'assainissement par ébullition, même si le temps d'ébullition diffère d'un foyer à l'autre et même d'un jour à l'autre. Notons que seulement 68,6 \% des répondants se fient à la qualité de l'eau distribuée par la STE.

Globalement, 57,9 \%des répondants considèrent que l'eau dont ils disposent pour leur consommation est le plus souvent de mauvaise qualité. De plus, ils sont en mesure d'associer à cette qualité une temporalité. $56 \%$ des répondants disent que l'eau des puits de la partie nord de la ville a un goût plus salé, ce qui est logique puisque ses eaux subissent des contaminations souterraines à partir des roches. $36 \%$ d'entre eux estiment en effet que la qualité de l'eau des puits et pompes est médiocre en saison des pluies. Ceci est également logique puisqu'en saison humide d'une part les puits sont contaminés par les pluies et les eaux de débordement des rivières, par pénétration d'eau de surface, et d'autre part les nappes sont contaminées par les eaux polluées, de surcroît riches en sédiments de la rivière (pollution par les sols). Les habitants qui utilisent l'eau des vendeurs d'eau attribuent, eux, la mauvaise qualité temporaire de l'eau à des problèmes techniques. Les habitants possèdent également un avis sur les conséquences sanitaires potentielles de leurs pratiques. En effet, 72,5 \%des répondants pensent que boire une eau impropre peut provoquer des maladies intestinales, notamment diarrhées et choléra. Seulement 3,1\% des répondants pensent ne pas courir de risque en buvant une eau impropre. Notons que même ceux qui citent des maladies non hydriques (maladies respiratoires) pensent pouvoir être contaminés par l'eau et du coup devraient logiquement y prêter attention.

Les discours des 1516 répondants traduisent ainsi d'une part une conscience de la mauvaise qualité de l'eau consommée et de la temporalité des fluctuations de qualité, d'autre part des connaissances solides sur les conséquences sanitaires potentielles de pratiques à risques. Cette bonne perception des risques est donc en inadéquation avec les pratiques quotidiennes observées.

\subsection{4- Perception de la menace sanitaire liée à l'eau stagnante et aux moustiques}

Les enquêtés pensent à près de $88 \%$ que la présence de déchets et l'insalubrité du quartier attirent les moustiques. $49 \%$ citent également la présence d'eau sale stagnante. $37 \%$ des répondants connaissent au moins deux des trois règles à respecter pour lutter contre les moustiques, bien que les comportements aient révélé qu'ils ne les appliquaient pas systématiquement.

Ensuite, près de $41 \%$ des répondants savent que les moustiques peuvent transmettre des maladies et parmi eux 62 \%citent les symptômes.

Ainsi, les comportements dangereux parfois observés ne peuvent s'expliquer par une perception faible des menaces liées aux moustiques ou des moyens de protection. Les connaissances des répondants dans ce domaine sont assez solides.

\subsection{5- Représentation d'exposition aux maladies de la population}

Plusieurs maladies sont citées par les répondants. Les maladies les plus fréquentes et auxquelles ils se sentent les plus exposés (tableau 43) sont le paludisme, la typhoïde et la diarrhée, avec plus de $63 \%$ de citations. Ces maladies sont bien liées globalement à l'eau et aux déchets. Ces choix sont reflètent la bonne perception de la menace à laquelle ils sont exposés. 
Tableau 3. Sentiment d'exposition aux maladies

\begin{tabular}{|l|l|}
\hline Sentiment d'exposition aux maladies & Fréquence \\
\hline Paludisme & 83,3 \\
\hline Diarrhée, maladies intestinales & 65,7 \\
\hline Grippe et toux & 32,5 \\
\hline Poliomyélite & 18,5 \\
\hline Maladies de peau & 36,7 \\
\hline Typhoïde & 64,5 \\
\hline Aucune maladie & 30,0 \\
\hline Ne sait pas & 27,5 \\
\hline autres & 19,2 \\
\hline
\end{tabular}

Source : enquêtes ménages 2016

\subsection{6- Perception de la maladie par la population abéchoise}

Dans le cadre ce travail, l'interprétation de l'épisode morbide est prise en compte après plusieurs jours de maladie, le plus souvent après la pratique de soins et la guérison du malade. La perception de la maladie peut donc avoir été influencée par le déroulement de l'itinéraire thérapeutique : elle ne correspond pas nécessairement à la lecture première qui a guidé les choix thérapeutiques. La perception de la nature et de la cause de la maladie constitue néanmoins une forte indication des normes d'interprétation des épisodes morbides et des schémas thérapeutiques qui leurs sont associés.

\subsubsection{1- Perception de la nature et de la cause de maladie}

A Abéché, les maladies sont fréquemment désignées en fonction de leurs manifestations, de leur cause présumée, de la technique thérapeutique utilisée ou de la période au cours de laquelle elle survient. Pour les ménages enquêtés, $100 \%$ ont affirmé avoir eu des cas des maladies dans leurs maisons, $72 \%$ des maladies liées à l'eau et $28 \%$ des maladies autres que les maladies liées à l'eau telles que la tuberculose, le SIDA, la rougeole etc. Ils définissent les maladies à partir de symptômes associés à près de $15 \%$ des cas : la présence de fièvre, le corps chaud, les vomissements, les yeux jaunis, les frissons, les maux de tête, les douleurs d'estomac et la diarrhée. La notification des épisodes morbides montrent qu'environ 42 $\%$ sont associées au paludisme, $33 \%$ aux maladies diarrhéiques et $12 \%$ aux dermatoses, $7 \%$ aux conjonctivites et $6 \%$ aux autres maladies telles que la fièvre jaune, les problèmes d'alimentation, les faiblesses liées à l'accouchement ou à l'allaitement, les allergies cutanées, les boutons, les plaies, les brûlures, les vers etc.

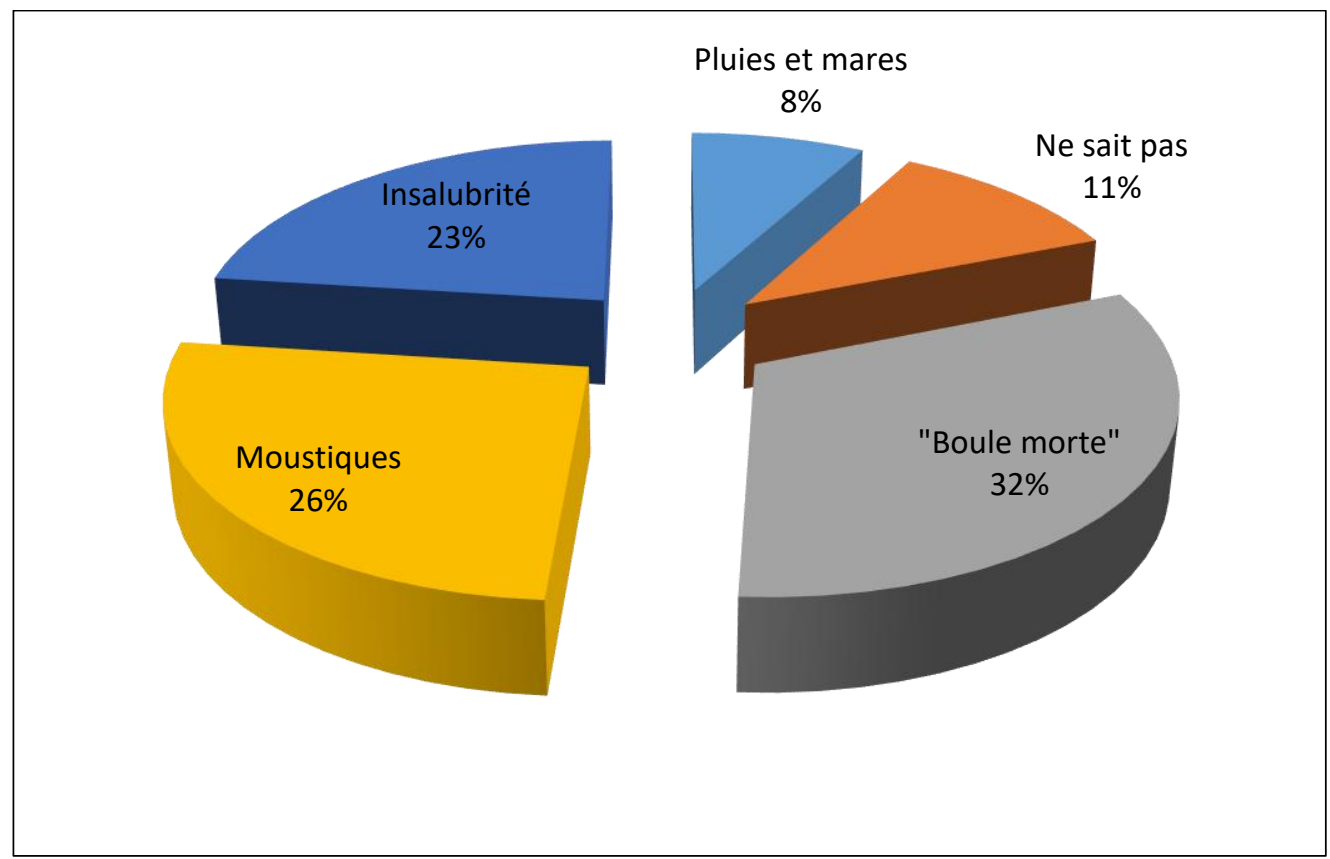

Source : enquête de terrain 2016

Figure 2. $\quad$ Perception de la nature de la maladie pour l'ensemble des interrogés

$10 \%$ des épisodes morbides sont définis par des symptômes difficiles à interpréter : le froid, les vertiges, les maux de côtes, les maux de poitrine, les problèmes respiratoires. L'interprétation donnée à la même maladie par les différentes personnes impliquées dans un épisode morbide est le plus souvent concordante, même si les personnes autres déclarent plus fréquemment ne pas savoir de quoi souffre le malade. La perception de la cause de la 
maladie donne lieu à des réponses très variées et souvent imagées. Près de $32 \%$ des épisodes morbides sont provoqués par la "boule morte" (les restes de nourriture de la veille), $23 \%$ l'insalubrité, $26 \%$ les moustiques, $8 \%$ par les pluies et les mares stagnantes et $11 \%$ autres (le sexe, l'alcool) (figure 34).

La saison des pluies est perçue comme une période accompagnée de maladies associées au paludisme et la dermatose, et est désignée dans $20 \%$ des épisodes morbides comme la cause des maladies liées à l'eau.

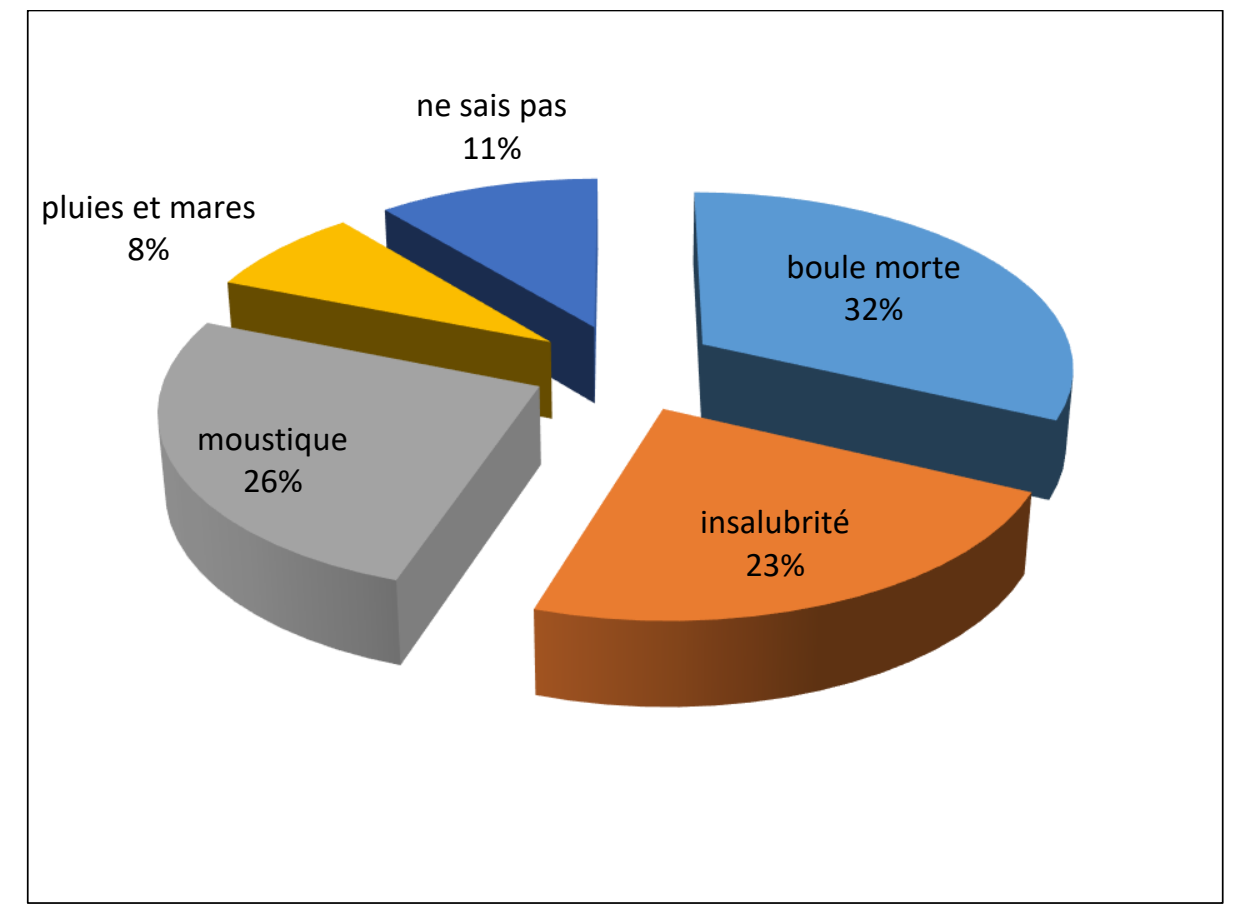

Source : Enquête de terrain 2016

Figure 3. Perception de la cause de la maladie pour l'ensemble des personnes interrogées

On note une grande difficulté de la population à expliquer l'origine des maladies enquêtées. Plus généralement, on souligne l'absence de repères forts pour la reconnaissance de la nature et de la cause de la maladie. Beaucoup trouvent leur explication dans la tradition ou la religion.

Le décalage entre la perception de la nature de la maladie et la cause qui lui est attribuée montre les différences dans les interprétations données par les enquêtés, l'interprétation des épisodes morbides s'effectue à travers les représentations individualisées à partir d'un savoir homogène et unifié. Par ailleurs, la tendance des répondants à adopter une posture fataliste face aux maladies liées à l'eau pendant la saison des pluies exprime une certaine impuissance face à une morbidité intense et récurrente.

La perception de la nature de la maladie varie fortement en fonction des caractéristiques symptomatiques de l'épisode morbide. Le profil symptomatique est moins directement associé à la perception de la cause de la maladie. En particulier, il n'apparaît pas de lien entre les symptômes et l'explication de la maladie.

\subsubsection{2- Influence de la nature et de la cause de la maladie}

Le diagnostic de la maladie est significativement associé au type et à la nature des soins pratiqués. Les maladies liées à l'eau tendent à être considérées comme ne nécessitant pas d'expertise externe car les épisodes morbides font l'objet d'une pratique plus fréquente de soins à domicile, mais d'une moindre propension à réaliser un ou plusieurs recours externes. Le diagnostic de ces maladies joue sur la consommation de médicaments dans le cadre des soins à domicile, notamment celle de paracétamol, Arthémeter et métronidazole, et favorise la pratique du "linge mouillé".

A l'inverse, les maladies liées à l'eau donnent lieu à une moindre utilisation d'aliments spéciaux et d'incantations ; elles réduisent le pourcentage des recours biomédicaux mais limitent significativement la consultation d'un thérapeute ou d'un médecin. La perception de la cause de la maladie est moins directement associée aux pratiques thérapeutiques qu'à la nature de la maladie. L'explication de la maladie par les symptômes influence relativement peu les comportements de 
consultation hors de la concession.

2.3- Perception, représentation et attitudes en matière de santé par la population (exemple du paludisme)

Les connaissances, les représentations et les attitudes en matière de santé influence sur les comportements de recours aux soins. Dans un premier temps, nous nous intéressons à la perception des caractéristiques du paludisme et les maladies diarrhéiques, en étudiant successivement la représentation de leurs causes, de leurs manifestations, de leurs gravités et des soins permettant de les traiter. Dans un second temps, nous avons étudié les attitudes en matière de santé en fonction des sources d'information en matière de santé, du rapport à la biomédecine et des stratégies de prise en charge de la maladie.

\subsection{1- Les connaissances des causes du paludisme}

L'étude des connaissances et des représentations associées au paludisme suppose, au préalable, un travail autour de la définition du paludisme. La population abéchoise utilise en effet une pluralité de dénominations pour désigner le paludisme, toutes comprises par l'immense majorité de la population. D'une manière générale, l'ensemble des fièvres associées aux vomissements sont appelées palu ${ }^{2}$. Ce terme nosologique renvoie à des définitions cliniques et des registres explicatifs.

Les personnes interrogées expliquent le plus souvent le paludisme par plusieurs causes (figure 36), 1,5 en moyenne, avec de sensibles distinctions dans les réponses données par les enquêté.

\footnotetext{
${ }^{2}$ Le mot palu est utilisé par les répondants pour désigner

le paludisme
} 


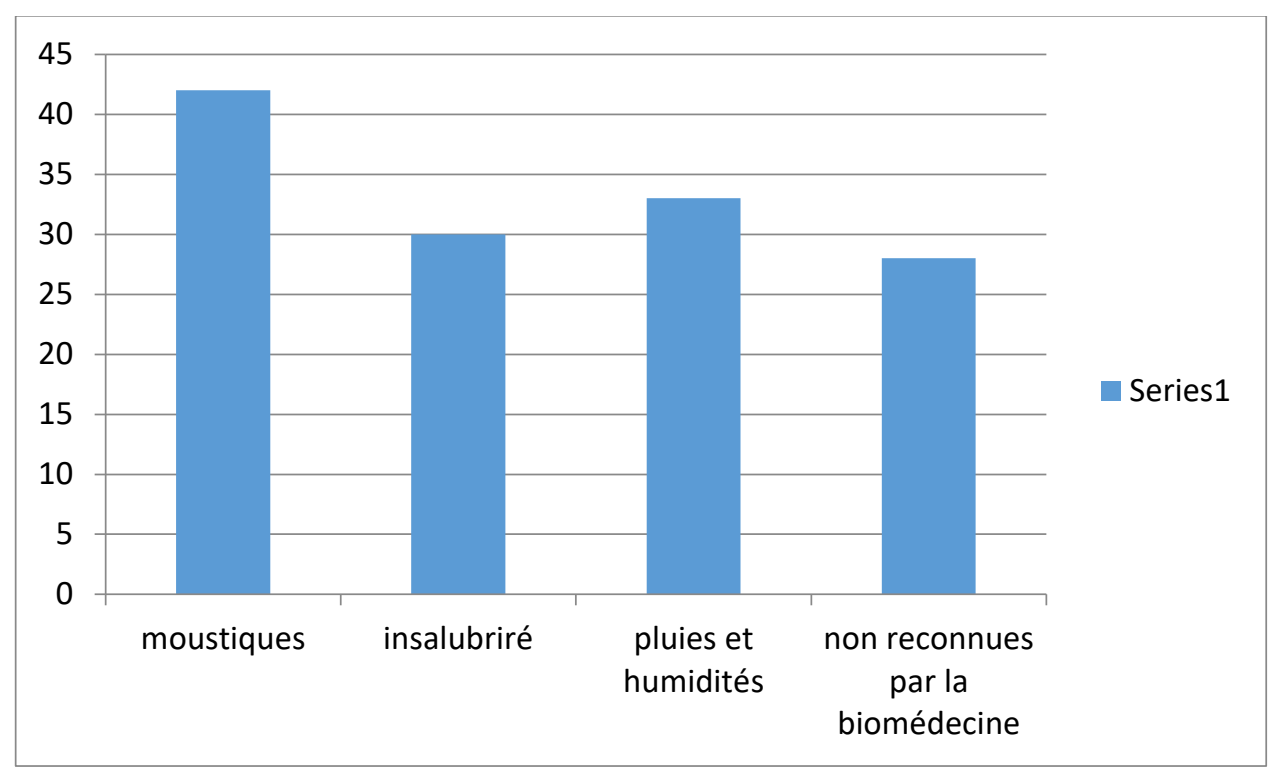

Source : Enquêté de terrain 2016

Figure 4. Causes perçues du paludisme par les ménages enquêtés (plusieurs réponses possibles par personne interrogée)

Les moustiques sont identifiés comme une cause de paludisme par près de $42 \%$ des personnes interrogées. Plus de la moitié des personnes considérant que les moustiques provoquent le paludisme l'associent également à une autre cause telle que la fraicheur et la boule morte ${ }^{3}$, le plus souvent des phénomènes environnementaux qui accompagnent ou favorisent indirectement la prolifération palustre.

Près de $30 \%$ des personnes interrogées considèrent le manque d'hygiène, et en particulier, la présence d'eau sale ou stagnante comme cause de paludisme. Un tiers des répondants explique le paludisme par une grande variété de facteurs associés à la période de l'année où survient la maladie : la tombée des pluies et l'humidité. $28 \%$ des personnes interrogées expliquent le paludisme par des causes non reconnues par la biomédecine : $40 \%$ par l'alimentation, les bains, la fatigue et $14 \%$ par le décalage chaud-froid. Le paludisme est avant tout considéré comme la volonté de Dieu.

Par ailleurs, une importante partie de la

${ }^{3}$ La boule morte est utilisée pour signifier le reste de la nourriture de la veille. En générale le mets principal dans la ville d'Abéché est le couscous (maïs, mil, sorgho etc.) qu'on met souvent dans une calebasse d'où le terme boule. population perçoit le lien entre le moustique et le paludisme. La majorité des interrogés ne maîtrise pas la notion de piqûres infectantes et ne comprend pas la véritable relation entre la présence d'eaux stagnantes et la prolifération des anophèles : « la relation entre la maladie et l'environnement est soupçonnée mais le mécanisme d'infection totalement ignoré $»$.

De manière attendue, les résultats montrent l'existence d'une association entre la connaissance du rôle des moustiques et la propension à faire un recours biomédical. Ainsi, lorsque l'enquêté explique exclusivement le paludisme par l'action des moustiques, il effectue le recours en structure sanitaire, y compris au cours des 48 premières heures de maladie.

Spécifiquement, la perception du paludisme favorise l'ingestion de médicaments, notamment de l'Arthemeter. A l'inverse, les représentations fatalistes de la maladie limitent la consommation de médicaments dans le cadre des soins à domicile et la propension à réaliser un recours biomédical.

En définitive, la perception de la cause de la maladie influence surtout la propension à réaliser un recours biomédical : inférieure à $23 \%$ dans les couples ignorant le moustique comme cause du paludisme, la consultation en structure sanitaire est supérieure à $35 \%$ dans les couples reconnaissant le rôle des moustiques, avec un niveau intermédiaire dans les autres ménages. Dans la même logique, la consultation rapide en structure sanitaire 
est 2,0 fois plus importante dans les couples considérant le moustique comme seule cause du paludisme que dans les couples fatalistes.

La perception de la cause de la maladie modifie peu les conditions de prise en charge du malade. Toutefois, les enquêtés connaissant le rôle du moustique semblent plus souvent décider en concertation que les autres et notamment ceux qui ignorent le moustique comme vecteur $\mathrm{du}$ paludisme. En outre, en l'absence de relation statistiquement significative, il apparaît que les enquêtés connaissant le rôle du moustique mobilisent des sommes plus importantes que les autres pour la réalisation du premier recours biomédical.

\subsection{2- La connaissance des symptômes du paludisme}

A Abéché, l'identification du paludisme repose sur un grand nombre de manifestations générales, au sein d'un univers de symptômes de faible gravité (figure 37). Le paludisme semble renvoyer à l'expression d'un trouble général non spécifique relativement bénin auquel est rattaché de nombreuses formes de dérangements fébriles survenant au cours de l'hivernage (Franckel A., 2004).

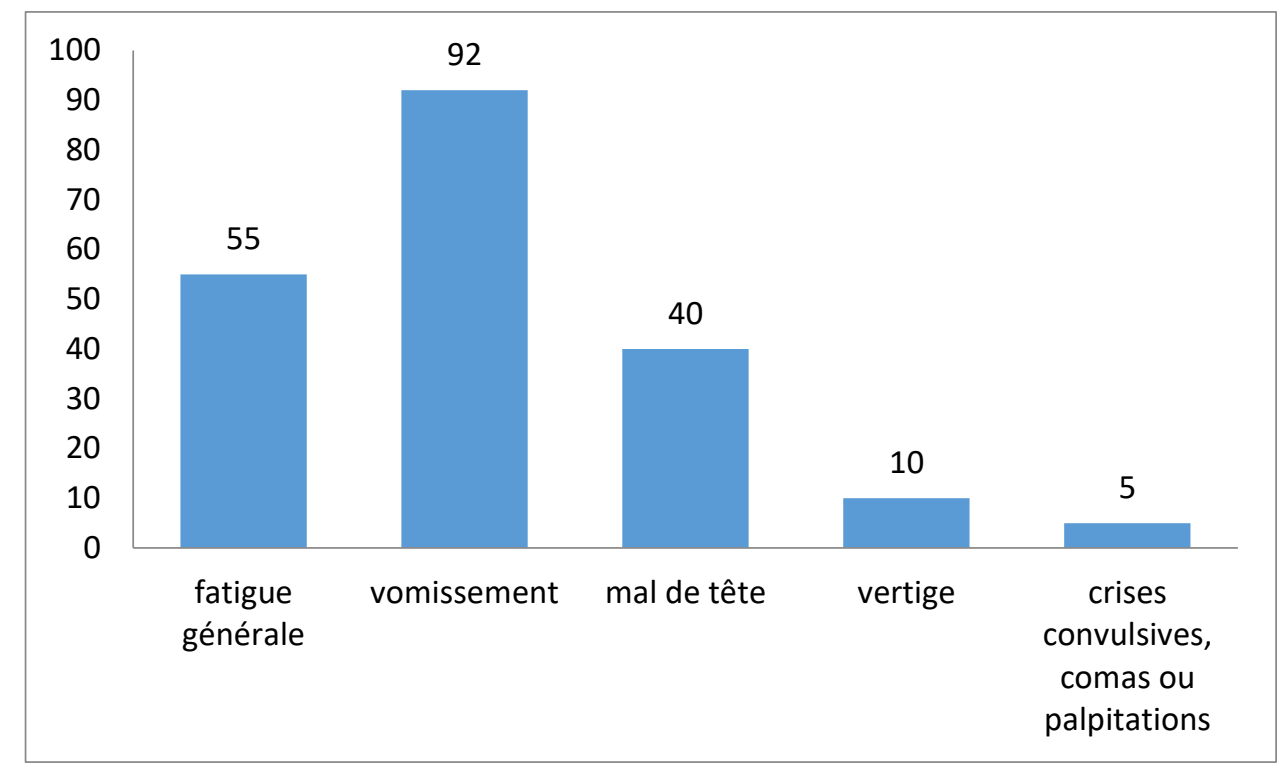

Source : enquête ménage 2016

Figure 5. Symptômes perçus du paludisme par les enquêtés (plusieurs réponses possibles par personne interrogée)

Le symptôme principal de reconnaissance du paludisme est l'hyperthermie, accompagnée de frissons (figure 39). A ce symptôme On associe plusieurs manifestations cliniques qui soulignent la dimension cette catégorie nosologique. Pour $55 \%$ des interrogés, le paludisme se décrit par un état de fatigue général induisant de l'inappétence, l'alitement, des bâillements et des étirements. Le mal de tête, expression d'un état de mal être, et les problèmes d'ictères, des yeux rougis ou larmoyants, sont associés au paludisme par plus de $40 \%$ des personnes interrogées.

D'une manière générale, la population perçoit le paludisme en plusieurs symptômes disjoints et sans lien évolutif direct : les manifestations du paludisme lui sont rarement associées, mais rattachées à d'autres maladies. Ainsi, $92 \%$ des répondants considèrent les vomissements comme symptôme du paludisme; moins de $10 \%$ lui associent les vertiges, les urines foncées et les cycles d'améliorations et de rechutes; de manière très marquée, seule une infime minorité, représentant moins de $5 \%$ des interrogés, associe au paludisme les crises convulsives, les comas ou les accès de palpitations.

Dans la mesure où la perception de l'origine du paludisme n'englobe pas les signes de paludisme, alors en cas d'évolution grave de l'épisode morbide, la population tend à réinterpréter la maladie en opérant un changement. On considère le paludisme en ce moment comme une maladie surnaturelle. On l'attribue à la sorcellerie et ou à l'envoutement. Le paludisme n'est le plus souvent pas considéré comme une maladie grave et mortelle, mais comme une maladie simple et quotidienne.

Le découpage du paludisme en plusieurs catégories étiologiques, construit la perception du paludisme comme une maladie peu dangereuse, ce qui pose la question de la notion de gravité du paludisme. Le plus souvent, la gravité du paludisme est perçue à travers des signes de fatigue générale. Pour près de $35 \%$ des interrogés, le principal critère de gravité du paludisme est 
la durée des symptômes classiques et, pour plus $75 \%$ d'entre eux, les signes généraux d'affaiblissement de l'organisme. $18 \%$ des interrogés perçoivent les vomissements comme un signe de gravité et moins de 23 $\%$ associent les crises convulsives, le coma et les palpitations cardiaques à un état du paludisme aggravé.

La connaissance des symptômes du paludisme et de ses signes de gravité est peu associée à la pratique des soins à domicile. Par contre, les déterminants des comportements de recours aux soins, la connaissance, relative, des symptômes du paludisme tendent à favoriser l'automédication. Ainsi, lorsqu'on associe le paludisme à l'une des principales manifestations du paludisme (fièvre, frissons, vomissements, céphalées, convulsions ou coma), alors l'on fait directement recours à l'automédication (100\% des enquêtés). L'influence de la connaissance des symptômes sur les comportements thérapeutiques se révèle cependant complexe; l'association de la gravité du paludisme aux vomissements, aux crises convulsives ou au coma favorise une consultation plus fréquente des thérapeutes traditionnels.

\subsection{3- Le traitement du paludisme}

Le paludisme est avant tout perçu comme une maladie naturelle, il apparaît tout à fait logique de constater que, pour son traitement, la population plébiscite les soins biomédicaux. $30 \%$ des répondants considèrent que l'offre de soins médicale est la plus efficace pour soigner le paludisme, les structures sanitaires, les injections et les comprimés sont respectivement cités par $23 \%, 16 \%$ et $18 \%$ des interrogés.

$48 \%$ des interrogés considèrent que les soins traditionnels pratiqués à domicile ou par des guérisseurs sont efficaces pour soigner le paludisme. Cette proportion est favorisée par la religion $(87 \%$ des enquêtés étant musulmans). La population se tourne d'abord vers les marabouts et les tradipraticiens avant de faire recours aux autres systèmes de soins. La lecture de l'efficacité des différents traitements doit être remise dans la perspective de la perception naturelle et plutôt bénigne du paludisme. Il est considéré comme normal qu'une personne soit atteinte du paludisme pendant la saison des pluies et la période de fraicheur, mais il est également perçu comme normal que le malade guérisse, même s'il n'est pas soigner, dans la mesure où le paludisme n'est pas considéré comme une maladie dangereuse. Les modes préférés de traitement du paludisme par la population dans les structures sanitaires sont la perfusion et l'injection. Les consultations en structure sanitaire n'ont d'intérêt que si elles débouchent sur la prescription d'une perfusion ou d'une injection. L'attrait des perfusions et des injections aux yeux de la population s'explique par la visibilité et la rapidité de leur action sur l'organisme. Dans les structures sanitaires étudiées, les injections des antis malarias (Arthémeter, Quinine, Palujet etc.) sont fréquentes et reflètent la pression exercée par la population auprès du personnel soignant.

Paradoxalement les perfusions et les injections font cependant également l'objet de représentations très négatives pour certaine population. Une part importante de la population y est réticente et près de $12 \%(\mathrm{n}=555)$ des interrogés déclarent ouvertement refuser que leurs malades surtout les enfants reçoivent une injection. La stigmatisation de l'injection intramusculaire repose sur plusieurs types de discours. Certains soulignent les risques, tout à fait réels, d'abcès pouvant entraîner une paralysie de la jambe en cas de piqûre mal injectée. Dans une logique conservatrice, une part importante des thérapeutes traditionnels affirme que les injections sont non seulement inefficaces pour traiter le paludisme, mais qu'elles rendent également inopérants les soins traditionnels. Enfin des rumeurs assurent que les injections peuvent réduire la capacité à procréer ou que, pratiquées au cours de la poussée dentaire, elles risquent d'entraîner le retournement les dents. Les représentations de l'efficacité des différents soins sont fortement associées aux comportements thérapeutiques. La perception d'un soin comme efficace induit une pratique nettement plus fréquente.

Lorsque les thérapeutes traditionnels sont déclarés compétents pour traiter le paludisme, leur consultation est plus fréquente que celle des structures sanitaires. A l'inverse, lorsqu'on perçoit l'efficacité des structures sanitaires pour le traitement du paludisme on consulte plus les structures sanitaires.

Pour près $60 \%$ des enquêtés fréquentant des structures sanitaires (68\% des enquêtés), la consultation en structure sanitaire est perçue comme moins chère dans les centres de santé publiques et l'Hôpital Régionale que dans les cabinets de soins et les cliniques privés. Ceci entraine une consultation dans le premier cas de la population démunie et dans le second cas des populations nanties. Cependant, pour près de $40 \%$ des interrogés, la différence entre le coût d'une consultation en structure sanitaire et auprès d'un guérisseur reste limitée ou soumise à des variations.

\section{CONCLUSION}

L'étude des connaissances, des perceptions et des attitudes en cas des épisodes morbide a mis en évidence un paradoxe majeur, la population d'étude se caractérise par des représentations et des attitudes en matière de santé très hétérogènes. A l'encontre d'interprétations simplistes ou idéologiquement marquées, la perception des maladies 
liées à l'eau reposent avant tout sur les enseignements d'une profonde expérience de ses caractéristiques médicales et épidémiologiques. Cependant, ces maladies restent mal connue à Abéché. En dépit de leur incidence majeure sur la morbidité et la mortalité, la perception de leurs causes, de leurs manifestations et de leurs traitements est le plus souvent approximative et fait l'objet de multiples amalgames.

La majorité des répondants a une conscience générale du rôle des vecteurs des maladies liées à l'eau, mais il apparaît dès lors logique que la population fasse appel à des explications liées à l'environnement, à la sorcellerie, aux tabous ou à des facteurs individuels de type nutritionnels pour expliquer la morbidité. Par ailleurs, la perception des moyens médicaux, bien que très spécifique, nous semble plutôt rationnelle. En effet, la population adhère pleinement aux principes de la médecine traditionnelle. Enfin, la population fait preuve d'une grande résignation face au paludisme qui est perçu comme un mal incontournable. Là encore, cette perception nous semble relativement logique au regard de l'expérience des habitants.

L'existence de multiples confusions sur la nature, les causes et le traitement des maladies liées à l'eau s'explique avant tout par la sous-exposition de la population à une information complète, relayée par un personnel qualifié. Les conditions de délivrance des messages sanitaires favorisent une compréhension parcellaire et déformée. A travers le prisme des expériences individuelles, le brouillage des informations sanitaires favorise au contraire la constitution de multiples syncrétismes, où les croyances sont superposées et imbriquées au sein de modèles de représentations multiformes (Espino, 1997 ; Ruebush, 1992). A Abéché, la population ne possède pas de modèle global et exclusif d'appréhension de la maladie car c'est un milieu soumis à l'évolution rapide des institutions traditionnelles, à une forte migration circulaire et à la diffusion de l'idéologie mouride, la circulation d'informations pseudo-médicales issues de tous horizons favorise l'élaboration de syncrétismes. Dès lors, les pratiques thérapeutiques ne peuvent plus être exclusivement considérées comme l'aboutissement d'un processus complet allant de l'interprétation au soin. La forte utilisation de l'automédication est bien plus le fruit de l'expérience des effets des différents traitements sur les symptômes que l'expression d'une complète adhésion au modèle biomédical (Hausmann Muela et al., 1998).

De manière attendue, la propension à consulter en structure sanitaire est directement associée à l'offre de soins biomédicale. Les stratégies thérapeutiques influencent également les comportements de recours aux soins. Dans la mesure où la non planification des dépenses de santé réduit la capacité des ménages à consulter en structure sanitaire, il apparaît intéressant, dans un environnement caractérisé par l'absence de toute forme de sécurité sociale, de mener une réflexion autour d'initiatives collectives permettant d'assurer l'anticipation d'un budget de santé par des cotisations régulières auprès des structures de santé.

\section{REFERENCES}

[1] AMAT-ROZE J.-M., REMY G., 1984. Paysage épidémiologique du paludisme dans l'espace ivoirovoltaïque. In DOUMENGE J.-P., dir., De l'épidémiologie à la géographie humaine. Talence: CNRS-CEGET, coll. «Travaux et documents de géographie tropicale», $\mathrm{n}^{\circ} 48, \mathrm{p}$. 97-108.

[2] Dombor Djikoloum Dingao, 2020, «Etude géographique des risques sanitaires liés à l'eau dans la ville d'Abéché au Tchad ». thèse de Doctorat $\mathrm{PhD}$, Université de Ngaoundéré $436 \mathrm{p}$

[3] Dombor Djikoloum Dingao et Djebé Mbaindogoum, 2019, «problématique de l'accès à l'eau potable dans la ville d'Abéché au Tchad ». Annales de l'Université de Moundou, Série A - Faculté des Lettres, Arts et Sciences Humaines, Vol.5 (2), janvier, ISSN 2304-1056

[4] Dombor Djikoloum Dingao, Tob-Ro N'Dilbé, René Joly Assako Assako, Michel Tchotsoua, 2019, «Etude des facteurs d'émergence du risque de maladies diarrhéiques dans la ville d'Abéché au Tchad». Revue Espace, Territoires, Sociétés et Santé, Université Felix HouphouetBoigny, juin

[5] Dombor Djikoloum Dingao et Mbainaimou Laokein Néhémie, 2019 «Dynamique territoriale, compétition foncière et approvisionnement en eau dans a ville d'Abéché ». cahier foncier au Tchad, vol.2(1), juin, ISSN 2077-0884

[6] Dombor Djikoloum Dingao et Djébé Mbaidonguem «Mutations socio-spatiales et approvisionnement en eau dans les quartiers périphériques d'Abéché (Tchad) » in Eau et Développement en milieu soudano-sahélienne d'Afrique : Analyse environnementale, sanitaire et géopolitique. Boniface Ganota, Daniel Valerie Baska Toussia et Natali Koussoumna Liba'a, Novembre 2019, ISBN 978-9956-74527-8.

[7] Ibrahima Sy, Mouhamadou Koita, Doulo Traoré, Moussa Keita, Baidy Lo, Marcel Tanner et Guéladio Cissé, «Vulnérabilité sanitaire et environnementale dans les quartiers défavorisés de Nouakchott (Mauritanie) : analyse des conditions d'émergence et de développement de maladies en milieu urbain sahélien », VertigO - la revue électronique en sciences de l'environnement [En ligne], Volume 11 Numéro 2 | septembre 2011, mis en ligne le 18 septembre 2011, consulté le 12 février 2021. URL: http://journals.openedition.org/vertigo/11174 ; DOI : https://doi.org/10.4000/vertigo.11174 
[8] Feachem R., Bradley D.J., Garelick H. and Mara D.D. 1980Health aspects of excreta and sullage management. Appropriate Technology of Water Suppl and Sanitation series. World Bank, Washington D.C.

[9] Gagliardi R. 1995- La formation scientifique et technique à l'usage des communautés traditionnelles .Perspectives. vol. $X X V ; n^{\circ} 1$, mars 1995

[10] Hughes C.C. et Hunter J.M. (1970) Diseases and "development" in Africa. Social science and medecine, 3 : 443-493.

[11] Hunter J.M., Rey L., Chu K.Y., Adekolu-John E.O. Mott K.E.- 1994- Parasitoses et mise en valeur des ressources hydriques. Un impératif: la négociation intersectorielle. OMS Genève 1994. 159 pp

[12] Issa Justin Laougué, Adbaye Béal Pureté, Dombor Djikoloum Dingao, Wakponou Anselme 2019, « Dynamique hydromorphologique, assainissement et érosion dans la ville d'Abéché ». Annales de l'Université de N'Djamena, Série A, Hors Série $N^{\circ} 1$, Decembre, ISSN 1991-0622

[13] Koné, Brama. Doumbia, Mohamed. Sy, Ibrahima. Dongo, Kouassi. Agbo-Houenou, Yveline. Houenou, Pascal Valentin. Fayomi, Benjamin. Bonfoh, Bassirou. Tanner, Marcel. Cissé, Guéladio. (2014) Étude des diarrhées en milieu périurbain à Abidjan par l'approche écosanté. VertigO. DOI: $10.4000 /$ vertigo. 14976

[14] Olivier J.L. et Le Jalle C.- 1998- Eau potable et assainissement dans les quartiers périurbains et les petits centres pS-Eau Coopération Française 157p.

[15] Parent G., Ouédraogo A., Zagré N.M. et al. Grands barrages, santé et nutrition en Afrique: au delà de la polémique. Cahiers Santé. 1997 ; 7, 417-22. 